\title{
Estimación de carga bovina localizada a partir de frecuencia de deposiciones en un pastizal de montaña
}

\author{
Axel R. von Müller ${ }^{1,4, \varpi ;}$; Ana M. Cingolani ${ }^{1,2}$; María V. Vaieretti ${ }^{1} \&$ Daniel Renison $^{3}$ \\ 1. Instituto Multidisciplinario de Biología Vegetal CONICET/UNC. 2. Facultad de Ciencias Exactas, Físicas y Naturales. \\ Universidad Nacional de Córdoba. 3. Centro de Ecología y Recursos Naturales Renovables Dr. Ricardo Luti. Universidad \\ Nacional de Córdoba-CONICET. 4. Instituto Nacional de Tecnología Agropecuaria EEA Esquel.
}

\begin{abstract}
Resumen. La cuantificación indirecta de la carga ganadera localizada en un determinado lugar dentro de un potrero puede ser una herramienta útil para estudiar los patrones de distribución espacial y temporal del ganado. En este trabajo nos propusimos identificar un modelo para estimar la carga ganadera bovina localizada a partir de la frecuencia de deposiciones en el suelo. Además, determinamos cual es el período de tiempo previo de carga ganadera mejor reflejado en las deposiciones observadas en un momento dado. Cuantificamos los animales presentes en parcelas fijas en potreros bajo diferentes manejos ganaderos cada 10 días y durante 15 meses para obtener un valor de carga ganadera localizada. En las mismas parcelas evaluamos la frecuencia de deposiciones al final del período de 15 meses. La frecuencia de deposiciones se relacionó lineal y positivamente con las cargas ganaderas localizadas integrando diferentes períodos previos (entre 1 y 15 meses). El período previo de 12 meses, además de explicar una proporción razonable de la varianza $\left(R^{2}=0.49\right)$, presentó una distribución normal de los residuales y representa la unidad temporal de manejo. El modelo fue validado a escala de potrero utilizando datos de frecuencia de deposiciones en fechas y/o potreros no utilizados para su calibración. Concluimos que el modelo propuesto es un buen estimador de la carga bovina localizada del año previo.
\end{abstract}

[Palabras clave: herbivoría doméstica, patrones de distribución, manejo ganadero, heces, tasa de descomposición]

\begin{abstract}
AвSTRACт. Estimation of localized cattle stocking rate from dung frequency in a mountain grassland: Indirect quantification of localized stocking rate in a certain place within a paddock could be a useful management tool to study livestock spatial and temporal distribution patterns. In the present study, we aimed to adjusting a model to estimate localized cattle stocking rates on the basis of dung frequency. We also determined the previous time period of localized stocking rate that is best reflected by the dung counts. We quantified the animals grazing in paddocks with different livestock managements, every 10 days during 15 months, to obtain a value of localized stocking rate. Likewise, in the same plots dung frequency was evaluated after 15 months. Dung frequency was linearly, positive and significantly related to localized stocking rate for all of the previous periods considered (from 1 to 15 months). We considered a previous period of 12 months, a timing which is in accordance to temporal management decisions and the estimated model explained a reasonable proportion of the variance $\left(\mathrm{R}^{2}=0.49\right)$, and finally, had a normally distributed residuals. The model was validated at paddock scale by using dung frequency data from dates and/or paddocks not included in the model adjustment. We conclude that the proposed model based on dung frequency and the proposed methodology is a good indicator of localized cattle stocking rate of the previous year.
\end{abstract}

[Keywords: domestic herbivory, distribution patterns, livestock management, faeces, decomposition ratedomestic herbivory, distribution patterns, livestock management, faeces, decomposition rate]

\section{INTRODUCCIÓN}

La cuantificación de la carga ganadera localizada puede ser una herramienta muy útil para estudiar los patrones de distribución del ganado y sus efectos sobre el ecosistema. En sistemas con ganadería extensiva, la presión de pastoreo puede ser muy variable, incluso dentro de un mismo potrero (Bailey 1995; Barnes et al. 2008), y determina en gran medida los patrones de distribución de la vegetación y la biota asociada (Adler et al. 2001; Fuhlendorf \& Engle 2001; Cingolani et al. 2008a). Para estimar las cargas localizadas en

Instituto Nacional de Tecnología Agropecuaria EEA Esquel. Chacabuco 513, (9200) Esquel, Chubut, Argentina. axelvm@gmail.com distintas áreas de un mismo potrero se puede apelar al conteo directo de animales (Senft et al. 1983; Bailey 1995), o más recientemente a la ayuda de dispositivos de geoposicionamiento adosados a los animales (Bertiller \& Ares 2008; Swain et al. 2011). Estas metodologías permiten conocer muy bien la heterogeneidad en la distribución del ganado pero exigen mucho tiempo y esfuerzo de trabajo a campo o bien son muy costosas, y se pueden utilizar en un número reducido de animales. También es posible inferir patrones de distribución de los herbívoros mediante indicadores indirectos como las deposiciones fecales sobre el suelo

Recibido: 12 de marzo de 2012; Fin de arbitraje: 20 de abril; Revisión recibida: 1 de junio; Aceptado: 3 de julio. 
(Cingolani et al. 2002; Tate et al. 2003). Si bien estos indicadores hasta el momento no han sido utilizados para cuantificar cargas localizadas, han servido para categorizar el uso espacial de ambientes dentro de un potrero (Teich et al. 2005; Kohler et al. 2006).

Las deposiciones de los herbívoros se descomponen a una tasa relativamente baja debido a su contenido elevado de fibra y lignina (van Soest 1994; Aerts 1997) y, por lo tanto, permanecen sobre el suelo durante períodos de tiempo prolongados (Tate et al. 2003). En regiones de clima templado o frío, las deposiciones son fácilmente identificables en el suelo hasta uno o dos años después de ser deyectadas (Cingolani et al. 2002; Dimander et al. 2003; Vaieretti 2010). Esto permite que a medida que transcurre el tiempo se vayan acumulando sobre el suelo. Es por ello que la cantidad de heces detectables en un determinado momento obedece a un balance entre la tasa de deyección (que depende de la carga localizada) y la tasa de descomposición. Bajo un mismo clima se puede asumir que la tasa de descomposición de heces no varía de manera substancial entre sitios, dado que se trata siempre del mismo material (Swift et al. 1979). Si bien la aplicación de antiparasitarios comerciales puede afectar tanto la tasa de deyección como la de descomposición de heces (Sommer \& Bibby 2002), en general se aplican de manera similar a todos los animales de una región (comunicación personal de veterinarios y productores). Es de esperar, entonces, que las deposiciones presentes en el suelo den una idea clara de la presión de pastoreo localizada de un determinado lugar y durante un período de tiempo previo (Cingolani et al. 2002; Teich et al. 2005). En línea con esto, White et al. (2001) encontraron que la abundancia de las deposiciones en sistemas de pastoreo intensivo está muy correlacionada con la proporción de tiempo que los animales permanecen en un área determinada.

Por esta razón, la combinación de una metodología de cuantificar las deposiciones en el suelo con la observación directa del ganado a campo (o el uso de dispositivos de geo-posicionamiento) permite ajustar modelos matemáticos para estimar-de manera indirecta- la carga ganadera localizada de un cierto período de tiempo previo. El uso de la abundancia de deposiciones comoindicador de presión de pastoreo localizada se ha aplicado con buenos resultados en diferentes estudios. Senft et al. (1983) modelaron los patrones espaciales de distribución del ganado bovino en las Planicies Centrales de Colorado a través del seguimiento de animales, y validaron luego los modelos mediante el uso de la densidad de deposiciones fecales. En la Sierra Nevada de California se identificaron y modelaron los factores asociados a los patrones de distribución del pastoreo bovino mediante la evaluación estacional de la deposiciones fecales (Tate et al. 2003). En los pastizales de Tierra del Fuego, Cingolani et al. (2002), a través de la intensidad de deposiciones, determinaron la selectividad de ovinos por paisajes en los que predominan los céspedes de pastoreo. En las Sierras Grandes de Córdoba, una primera aproximación en el tema fue realizada por Teich et al. (2005), quienes utilizaron tanto la observación directa de animales como la frecuencia de deposiciones para diferenciar sitios con alta y baja carga. Sin embargo, en ninguno de estos trabajos se estableció una relación cuantitativa entre la abundancia de deposiciones y la carga ganadera localizada.

En general, los estudios que consideran la intensidad de uso ganadero (carga o presión ganadera) como un factor determinante de respuestas ecosistémicas, utilizan una estimación cualitativa de la presión de uso. Por ejemplo, dos, tres, o a lo sumo cuatro situaciones diferentes de presión ganadera (Teich et al. 2005; Cingolani et al. 2005; Kemp \& Michalk 2007). Además, a menudo en este tipo de estudios se supone que la carga ganadera presente en un determinado potrero se traduce en una presión de pastoreo homogénea sobre toda su superficie. Sin embargo, la distribución de los animales dentro de un potrero suele ser muy heterogénea, lo cual resulta en diferente intensidad de carga/uso en diferentes áreas del potrero (Adler et al. 2001; Fuhlendorf \& Engle 2001; von Müller 2011). Es por ello que la incorporación de modelos cuantitativos que relacionen las deposiciones con la carga localizada puede facilitar la evaluación local de los efectos puntuales de la presión ganadera sobre el ecosistema, y potenciaría la utilización de resultados para hacer inferencias teóricas, comparar con otros ecosistemas, o aplicar al manejo.

El objetivo general de este trabajo fue ajustar una metodología indirecta de estimación de carga ganadera bovina a partir de la frecuencia de deposiciones fecales presentes en el suelo. Para ello nos propusimos los siguientes objetivos específicos: 1 ) detectar cuales son los períodos de carga ganadera previa localizada que mejor se reflejan en las deposiciones presentes en un momento dado, 2) obtener uno 
o más modelos estimativos de carga ganadera localizada a partir de una metodología estándar que integren un determinado período de tiempo previo al momento de la evaluación, y 3) validar dichos modelos para otras áreas y/o fechas, en la misma región, y seleccionar el más adecuado.

\section{MATERIALES y MÉTODOS}

\section{Área de estudio}

El estudio se realizó en el piso superior de las Sierras Grandes de Córdoba, dentro del Parque y Reserva Nacional Quebrada del Condorito. El clima es templado frío, y a los 2100 m.s.n.m., altitud aproximada del área de estudio, la temperatura media anual es cercana a $\operatorname{los} 8^{\circ} \mathrm{C}$. La temperatura media del mes más cálido es de $11.4^{\circ} \mathrm{C}$, mientras que la del mes más frío es de $5^{\circ} \mathrm{C}$, pero con mínimas absolutas de hasta $-20{ }^{\circ} \mathrm{C}$. Pueden producirse heladas en cualquier momento del año (Cabido 1985). El promedio anual de precipitación es de 901 $\mathrm{mm}$, concentradas entre los meses de noviembre y abril (Colladón et al. 2010). Los suelos derivan de las rocas graníticas que forman el basamento, y junto con la ganadería determinan una gran heterogeneidad en la vegetación, conformando el mosaico característico de las Sierras Grandes de Córdoba. Este mosaico consiste en una combinación de pajonales finos, pajonales gruesos, céspedes y bosquecillos de Polylepis australis. Además, existen numerosos afloramientos graníticos y grandes extensiones de roca expuesta por erosión, formando pavimentos y pedregales (Cingolani et al. 2003, 2004). En el Parque Nacional, la ganadería se utiliza en algunos potreros para manejar el paisaje, evitando la excesiva acumulación de biomasa y la dominancia de pajonales en mata (Cingolani et al. 2010). Por su parte, en la Reserva Nacional la propiedad es privada. Para este estudio utilizamos potreros pertenecientes al Parque Nacional y a la Estancia Santo Tomás, en la Reserva Nacional (ver Anexos I \& II de Información Suplementaria en www.ecologiaaustral.com.ar).

\section{Diseño del estudio}

Para el ajuste de los modelos de carga ganadera localizada utilizamos siete potreros. Dos potreros con manejo continuo del pastoreo (C2 y C8) y cinco potreros bajo sistemas de pastoreo rotativo estacional (sistema R1, con tres potreros en rotación; y sistema R2, con dos potreros en rotación; ver Anexos I \& II). Elegimos diferentes sistemas de pastoreo con el objetivo de tener más variabilidad en el tipo de uso de los potreros, de forma que el modelo estimativo sea aplicable a distintos tipos de manejo, incluyendo situaciones en las cuales el manejo se desconoce. La tasa de descomposición de las deyecciones bovinas en el área de estudio es lenta (Vaieretti 2010) ya que excede largamente los tiempos de descanso y permanencia del ganado en los potreros bajo sistema de pastoreo rotativo. Por esta razón, cabe esperar que las deyecciones reflejen un período de carga localizada relativamente largo e independiente del tipo de sistema de pastoreo implementado.

Mediante el uso de un Sistema de Información Geográfica del área (Cingolani et al. 2004, 2008b) seleccionamos un total de 149 parcelas de 1 ha $(100 \times 100 \mathrm{~m})$ distribuidas al azar estratificado por tipo de paisaje, dentro de los siete potreros elegidos para el ajuste de los modelos. Por la alta heterogeneidad a pequeña escala que presenta esta región, las parcelas generalmente incluyeron un mosaico de diferentes tipos de parches de vegetación y roca, representando a las diferentes unidades de paisaje presentes en el área (Cingolani et al. 2004).

\section{Registro de la carga ganadera localizada en las parcelas}

Dado que los grandes herbívoros en general (Senft et al. 1987), y los bovinos en particular (Bailey et al. 1996), cambian de unidad de paisaje aproximadamente una vez cada 10 días, decidimos utilizar esa frecuencia para registrar de forma visual la carga ganadera en el campo. Entre el mes de abril de 2007 y junio de 2008, el mismo observador hizo tres recorridos por mes, visitando la totalidad de las 149 parcelas durante el mismo día en cada recorrido. Los recorridos estuvieron separados por 7 a 13 días entre sí. Durante cada recorrido, estimó la carga bovina instantánea localizada de todas las parcelas, mediante conteos directos de la cantidad y categoría de bovinos presentes dentro de ellas. Luego, estos valores se transformaron en unidades ganaderas (UG), en las que las vacas, las vaquillonas y los novillos correspondieron a 1 UG, y los toros a 1.3 UG (modificado de Cocimano et al. 1975). El observador realizó los recorridos durante horas diurnas y comenzando siempre desde lugares diferentes para evitar sesgos en las observaciones en relación al horario de visita. Para evitar disturbar a los animales, el observador hizo el conteo con binoculares desde algún punto de observación alejado unos $300 \mathrm{~m}$ de las parcelas. Para los potreros con sistema de pastoreo rotativo estacional, el observador hizo los recorridos únicamente durante los períodos en los que había animales. Para las fechas en las que no había animales, registró como cero la carga localizada de cada parcela. En total, realizó 45 registros de cargas ganaderas localizadas en cada una de las parcelas durante el período de 15 meses que duró el estudio (tres registros por mes).

\section{Registro de la frecuencia de deposiciones}

En julio de 2008, es decir a los 15 meses del inicio de los recorridos, calculamos la frecuencia de deposiciones en las mismas parcelas en las que estimamos la carga ganadera localizada. Para ello registramos la presencia de deposiciones bovinas en un cuadrado de $30 \times 30 \mathrm{~cm}$ arrojado 250 veces, 
cada dos pasos consecutivamente a la derecha y a la izquierda de una transecta que zigzaguea íntegramente dentro de la parcela (Teich et al. 2005). Consideramos como válida toda presencia de heces identificable dentro del cuadrado, más allá del estado de degradación que presentara. Utilizamos esta metodología por poseer una regla de decisión sencilla y objetiva (presencia vs. ausencia). Luego, calculamos la frecuencia de deposiciones como el porcentaje de presencias respecto al total de veces que arrojamos el cuadrado.

\section{Determinación de sesgos y corrección de cargas localizadas}

Debido a que la observación de la carga localizada fue hecha desde lejos, presumimos que podía haber algún tipo de sesgo en las observaciones. Para analizar este posible sesgo, en primer lugar calculamos una carga promedio por mes para cada parcela (UG/ha) a partir de los tres datos mensuales obtenidos durante las observaciones de campo. Luego, para cada parcela promediamos los valores de carga observada durante los 15 meses que duró el estudio. Luego, a partir de los valores de todas las parcelas pertenecientes a un mismo potrero obtuvimos un valor medio para cada uno de los siete potreros en los que se realizó el ajuste (ver Anexo II). Este valor debería reflejar la carga ganadera del potrero. Por ello, estos valores fueron comparados, a través de una regresión simple, con las cargas informadas por APN y el productor privado para el mismo período (abril 2007 a junio 2008). Dado que los resultados de este análisis indicaron que existía un sesgo, utilizamos el modelo de regresión obtenido para corregir los valores mensuales de cargas obtenidos a través de la observación directa de animales. El nivel de detalle y control de los animales que tienen APN y el productor privado, permiten confiar en la corrección realizada. Para todos los subsiguientes análisis utilizamos el dato mensual por parcela corregido.

\section{Ajuste de los modelos}

Debido a que no sabíamos exactamente por cuánto tiempo son detectables las deposiciones, exploramos las relaciones entre la frecuencia de deposiciones y la carga ganadera localizada previa integrando diferentes períodos de tiempo. Para ello, a partir de los registros de cargas mensuales corregidas, calculamos para cada parcela las cargas medias de diferentes períodos previos a la medición de julio, desde un único mes previo (junio 2008) hasta 15 meses previos (abril 2007-junio 2008). Posteriormente, utilizando los datos de frecuencia de deposiciones por parcela tomados en julio de 2008 como variable independiente, hicimos 15 regresiones simples con las cargas localizadas integrando cada uno de los períodos (desde 1 mes hasta 15 meses previos), como variables dependientes. En todos los casos, calculamos los intervalos de confianza para la ordenada al origen y la pendiente. Además, para cada regresión, calculamos los residuales y evaluamos la normalidad con la prueba de Kolmogorov-Smirnov. Por otro lado, para analizar en cada caso si parte de la varianza no explicada podía deberse al manejo u otras características de los potreros, comparamos los residuales entre los diferentes potreros con un ANOVA de un factor.

Consideramos que el período de cargas mejor reflejado por las deposiciones se debe manifestar en las siguientes características de las regresiones: 1) una pendiente significativamente diferente de cero, 2) una ordenada al origen no diferente de cero, 3) residuales con distribución normal, 4) residuales que no difieran entre potreros, y 5) una varianza explicada similar o más alta que las regresiones con los demás períodos. Dado que ninguno de los períodos cumplió con la totalidad de las condiciones mencionadas, pre-seleccionamos los dos períodos que más se acercaban a cumplir con dichas condiciones, y utilizando estos períodos, obtuvimos dos modelos estimativos. Por último, para realizar una evaluación comparativa del ajuste de estos dos modelos calculamos la Raíz del Cuadrado Medio del Error (RCME; Grigera et al. 2007).

\section{Validación y comparación entre los dos modelos pre-seleccionados}

Para validar los modelos, es necesario contar con datos externos, no utilizados para el ajuste de los mismos (Sørensen 1990). A nivel de parcela de 1 ha, sólo disponíamos de datos externos de frecuencia de deposiciones, pero no de carga previa observada en el campo para esas mismas parcelas. Por eso, hicimos la validación de ambos modelos a nivel de potrero, incluyendo fechas $\mathrm{y} / \mathrm{o}$ potreros no utilizados para el ajuste (Anexos I \& II). Utilizamos de forma independiente cinco conjuntos de datos de frecuencia de deposiciones tomados en cinco fechas distintas: marzo de 2007, septiembre 2007, enero 2008, agosto 2008 y marzo 2009. Los datos de las tres primeras fechas fueron tomados en las mismas 149 parcelas de los siete potreros utilizados para el ajuste del modelo. Los datos de agosto de 2008 (una fecha prácticamente igual a la utilizada para el ajuste del modelo), fueron tomados en 270 parcelas de 1 ha seleccionadas con el mismo criterio que antes, pero distribuidas en 11 potreros diferentes, seis con manejo continuo del pastoreo $(\mathrm{C} 1, \mathrm{C} 3, \mathrm{C} 4$, C5, C6 y C7) y cinco en potreros manejo rotativo del pastoreo (R3a, R3b, R3c, Rt1 y Rt2). Finalmente, los datos de marzo de 2009 fueron tomados en las 419 parcelas $(149+270)$ repartidas en la totalidad de los 18 potreros.

Para cada una de las cinco fechas, utilizamos la frecuencia de deposiciones para estimar, por medio de los dos modelos lineales correspondientes, las cargas ganaderas bovinas previas de los dos períodos pre-seleccionados. A partir de estas cargas estimadas por parcela, obtuvimos los promedios por potrero de los 10 lapsos de tiempo (resultantes 
de la combinación de cinco fechas $\mathrm{x}$ dos períodos previos). Por otro lado, a partir de la información sobre la carga de los potreros suministrada por APN y el propietario privado, calculamos las cargas bovinas correspondientes a los mismos 10 lapsos. Después, para cada uno de los 10 lapsos realizamos una regresión lineal entre la carga promedio estimada mediante la frecuencia de deposiciones como variable independiente y la carga informada como variable dependiente. Por último, realizamos una regresión para cada uno de los dos períodos previos preseleccionados agrupando las cinco fechas. Para validar los modelos esperábamos que, para las cinco fechas por separado y para las cinco fechas agrupadas, la regresión fuese significativa y la varianza explicada fuese alta; que la ordenada al origen no difiriera significativamente de cero; y que la pendiente no difiriera significativamente de uno. Finalmente, teniendo en cuenta la comparación a través de la RCME y los resultados de la validación, seleccionamos el mejor modelo.

\section{Resultados}

\section{Determinación de sesgos y corrección de cargas localizadas}

La regresión entre las cargas promedio por potrero obtenidas de la observación directa y las obtenidas a partir de la información suministrada por APN y el productor privado resultó significativa y la varianza explicada $\left(\mathrm{R}^{2}\right)$ fue alta (Figura 1). La ordenada al origen no difirió significativamente de cero, pero la pendiente fue significativamente más baja que uno, lo que señala que durante las observaciones de campo, las cargas se sobreestimaron (Figura 1). Dado que la ordenada al origen no difirió de cero, forzamos la regresión al origen. De este modo, utilizamos la pendiente obtenida ( $b=0.596$; Figura 1$)$ para multiplicar por los datos mensuales calculados para las parcelas, obteniendo un dato mensual corregido.

\section{Selección de modelos}

La pendiente de la regresión lineal entre la frecuencia de deposiciones bovinas medida en julio de 2008 y la carga observada (corregida) durante los diferentes períodos previos fue significativamente diferente de cero en todos los casos $(P<0.001)$. La varianza explicada $\left(R^{2}\right)$ varió entre 5 y $61 \%$, con los valores más bajos para los períodos más cortos (Figura 2a). A partir de un período previo integrado de seis meses se alcanzó una meseta en la varianza explicada cercana al 50\%, que se mantuvo en valores similares hasta los 15 meses previos (Figura 2a). Por otro lado, el valor de la ordenada al origen de las regresiones

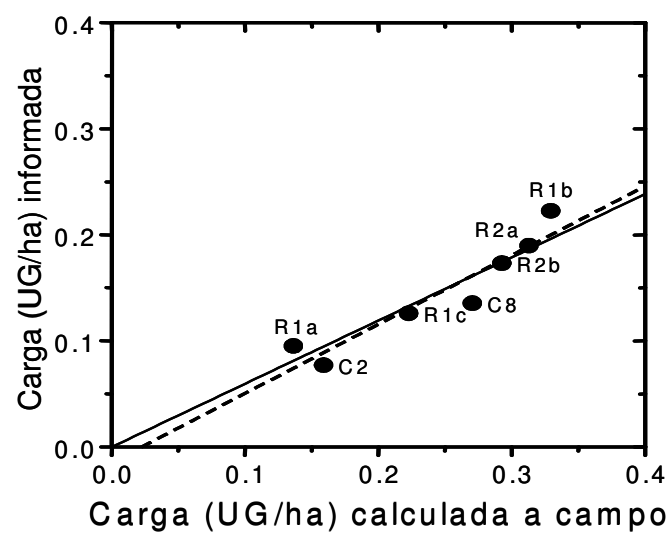

Figura 1. Cargas informadas (abril 2007-junio 2008) en relación a las estimadas a campo a partir del conteo directo de animales para los siete potreros en los cuales se realizó ajuste del modelo estimativo (círculos negros). La línea entrecortada indica la recta de regresión lineal: $\mathrm{Y}=0.651 \pm 0.264^{*} \mathrm{X}-0.015 \pm 0.053 ; \mathrm{R}^{2}=0.89 ; P \leq 0.05$. La línea sólida indica la regresión forzada a pasar por el origen: $\mathrm{Y}=0.596 \pm 0.065^{*} \mathrm{X} ; \mathrm{R}^{2}=0.88 ; P \leq 0.05$.

Figure 1. Informed stocking rates (April 2007-June 2008) in relation to field stocking rates as estimated from animal counts for the seven paddocks used for model adjustment (black circles). Details in legend in Spanish.

resultantes utilizando períodos entre 9 y 14 meses previos no difirió significativamente de cero, mientras que para períodos más cortos o más largos, la ordenada al origen fue más baja que cero (Figura 2b). En particular para 12 y 13 meses previos, la ordenada al origen fue muy cercana a cero. Además, para períodos muy cortos el estadístico $Z$ de KolmogorovSmirnov fue alto y significativamente distinto de lo esperado según una distribución normal (Figura 2c). Recién a partir de los cinco meses se estabilizó, con fluctuaciones alrededor del valor crítico. Para todos los períodos previos exceptuando los 9 meses, los residuales difirieron significativamente entre potreros (Figura 2d). Dado que ninguno de los períodos cumplió con la totalidad de las condiciones planteadas previamente, preseleccionamos los dos períodos que más se acercaron a cumplir con dichas condiciones: 9 y 12 meses previos. Dado que para ambas regresiones la ordenada al origen no fue significativamente diferente de cero, las repetimos forzándolas a pasar por el origen. De este modo obtuvimos dos modelos, uno para cada período preseleccionado, que evitan las estimaciones negativas.

El período de 9 meses previos fue preseleccionado porque fue el único para el cual los residuales no difirieron significativamente entre potreros, lo cual posibilitaría estimar las cargas de este período con el menor sesgo posible debido al manejo (Figura 2d). Sin embargo, este período tuvo 

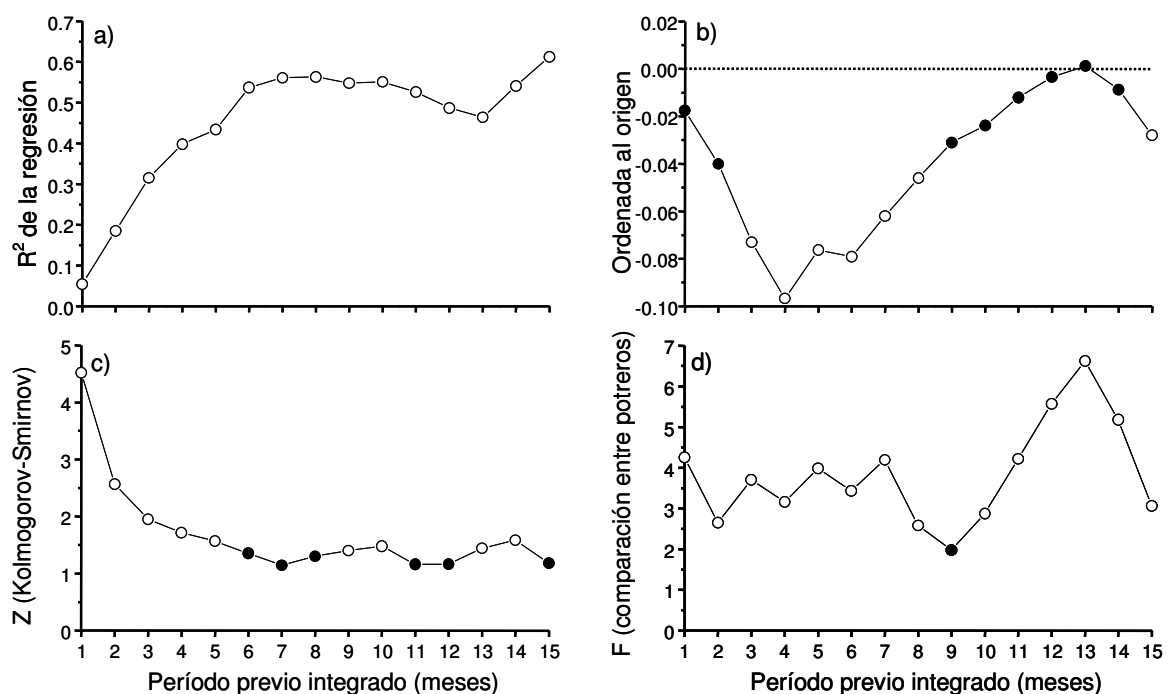

Figura 2. Variación de los parámetros de la regresión entre la frecuencia de deposiciones medida en julio de 2008 en función a la carga ganadera integrada de diferentes períodos previos (desde 1 a 15 meses). a) Varianza explicada $\left(R^{2}\right)$ $\circ=$ casos en los que la regresión fue significativa. b) Ordenada al origen, $\mathrm{O}=$ significativamente diferente de cero, $\bullet=$ no significativamente diferente de cero. c) Z de Kolmogorov-Smirnov indicando el grado de ajuste de los residuales a una distribución normal. $\odot=$ los residuales difirieron significativamente de una distribución normal, $\bullet=l o s$ residuales no difirieron de una distribución normal. d) valor del $F$ obtenido al comparar los residuales entre los diferentes potreros, $0=\operatorname{los}$ residuales difirieron significativamente entre potreros, $\bullet=l o s$ residuales no difirieron significativamente entre potreros.

Figure 2. Variation of the regression parameters between dung frequencies measured in July 2008 as a function of integrated stocking rate for different previous periods (from 1 to 15 months). a) Explained variance $\left(R^{2}\right)$, b) Intercept, c) $Z$ from Kolmogorov-Smirnov obtained indicating residual adjustment to a normal distribution, d) $F$ value obtained from the comparison among the residuals from the different paddocks under study. Details in legend in Spanish.

dos desventajas. Por un lado, los residuales de la regresión no ajustaron a una distribución normal (Figura 2c). Por otro lado, la ordenada al origen, si bien no difirió significativamente de cero (Figura $2 b$ ), tuvo un valor negativo lo bastante importante como para que al forzar la recta por el origen la pendiente cambiara de manera visible (Figura 3a). Cuando se forzó la recta por el origen, los resultados en cuanto a la normalidad de los residuales y la diferencia de los residuales entre potreros no cambiaron.

El período de 12 meses previos fue preseleccionado porque el valor de ordenada al origen resultó muy cercano a cero (Figura 2b), aunque la varianza explicada fue algo menor que la del modelo anterior (Figura 2a). Además, la distribución de los
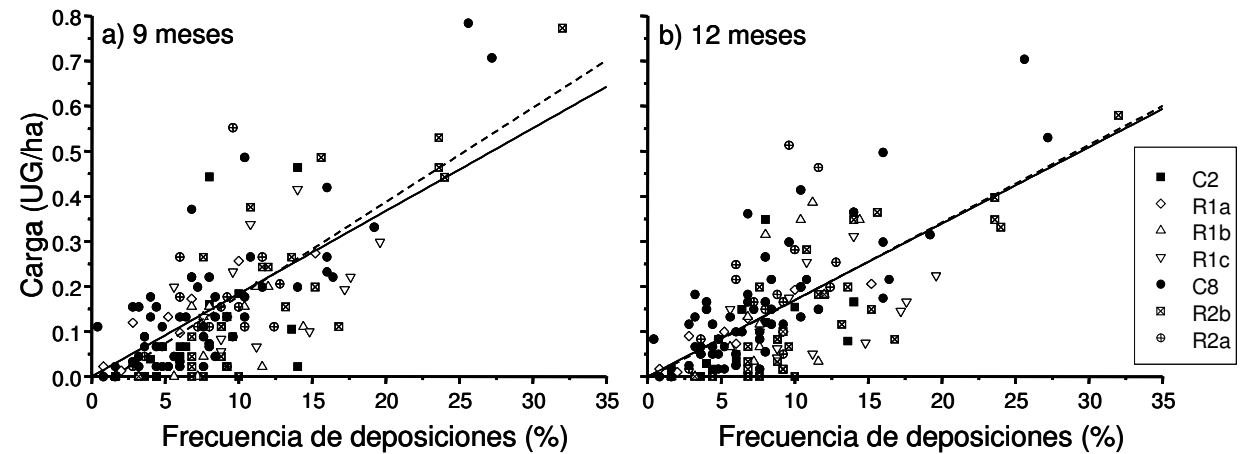

Figure 3. Carga promedio por parcela en función de la frecuencia de deposiciones medida en julio de 2008. En (a) la carga promedio integrada corresponde a los 9 meses previos, y en (b) a los 12 meses previos. Cada punto representa una parcela, y los diferentes símbolos indican los siete potreros utilizados para el ajuste de los modelos. Las líneas entrecortadas indican las regresiones sin ser forzadas por el origen. (a) $Y=-0.031 \pm 0.032+0.021 \pm 0.003^{*} X ; R^{2}=0.55 ; R C M E=0.1740$; $P<0.001 ;$ (b) $\mathrm{Y}=-0.003 \pm 0.030+0.017 \pm 0.003^{*} \mathrm{X}, \mathrm{R}^{2}=0.49$, $\mathrm{RCME}=0.1622 ; P<0.001$. Las líneas sólidas representan las rectas de las regresiones forzadas por el origen (a) $Y=0.018 \pm 0.002^{*} X ; R^{2}=0.54 ; R C M E=0.1002 ; P<0.001^{*} X$; (b) $Y=0.017 \pm 0.001^{*} X$; $\mathrm{R}^{2}=0.49 ; \mathrm{RCME}=0.0963 ; P<0.001$.

Figure 3. Mean stocking rate as a function of dung frequency measured in July 2008. In (a) the mean stocking rates corresponds to the 9 previous months, and in (b) to the 12 previous months. Details in legend in Spanish. 

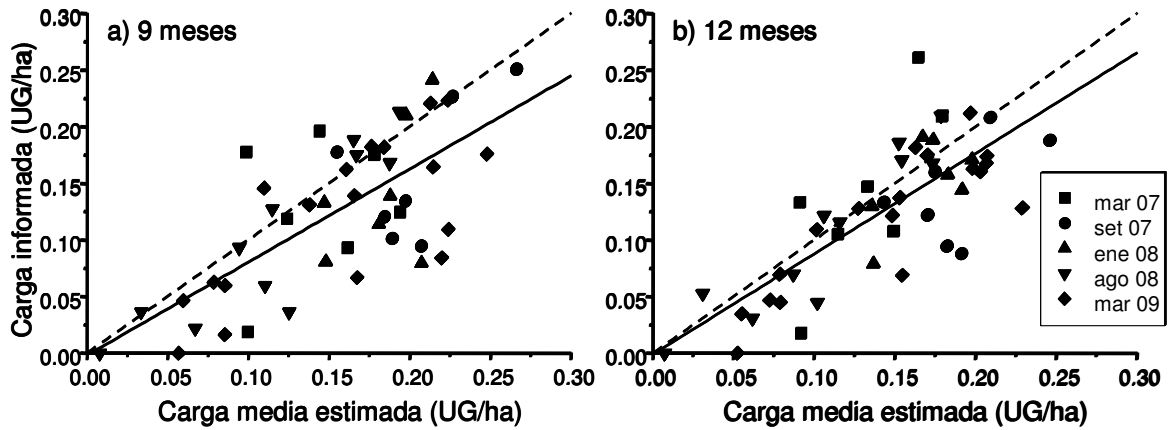

Figura 4. Carga media por potrero informada en función de la carga media estimada por los modelos para (a) 9 y (b) 12 meses, para todas las fechas agrupadas (ver detalles en Tabla 2). Cada punto representa un potrero cuya carga estimada se estimó a partir del promedio de cargas estimadas de sus parcelas y de la carga informada por los administradores. La línea entrecortada indica la relación 1:1; la línea sólida indica la recta de regresión en cada caso.

Figure 4. Relationships among mean informed paddock stocking rates and the estimated paddock stocking rate by using the (a) 9 and (b) 12 polled data models (see details in Table 2). Details in legend in Spanish.

residuales no difirió significativamente de la distribución normal (Figura 2c). Asimismo, es importante destacar que este período integra un año entero, que es la unidad temporal de manejo. Al tener una ordenada al origen muy cercana a cero, la pendiente permaneció casi exactamente igual al forzar la regresión a pasar por el origen (Figura 3b). El factor potrero explicó $18.7 \%$ de la varianza de los residuales de la regresión forzada por el origen, lo que significa $9.6 \%$ de la varianza total. Los potreros R1b y R2b tuvieron los residuales más negativos y difirieron significativamente del potrero R2a, que tuvo los residuales promedio más positivos. El resto de los potreros no difirió significativamente de ninguno de los demás (ver Anexo III). En los dos primeros casos se trató de potreros con sistema de pastoreo rotativo en los cuales el período de alta concentración de animales fue inmediatamente antes de hacer las mediciones de deposiciones (julio 2008), mientras que el período de descanso fue varios meses antes (ver Anexos I y III). En el caso del potrero con residuales positivos más altos, el patrón fue inverso, el período de descanso ocurrió inmediatamente antes de hacer las mediciones de deposiciones (julio 2008), mientras que el período de alta concentración de animales ocurrió varios meses antes (ver Anexo I). Esto indica que ante una misma carga anual promedio, se detecta más frecuencia de deposiciones cuando la medición se hace hacia el final del período de carga, que cuando se hace hacia el final del período de descanso.

La raíz del cuadrado medio del error (RCME) fue 0.174 y 0.1002 para las regresiones utilizando un período de 9 y 12 meses previos, respectivamente, y 0.162 y 0.096 para las regresiones forzadas por el origen utilizando los mismos dos períodos (Figura 3). Estos valores indican que el modelo de 12 meses forzado al origen es el que minimiza los errores de estimación.

\section{Validación de los modelos estimativos para bovinos}

Para ambos modelos pre-seleccionados (9 y 12 meses previos), las regresiones de validación hechas para las cinco fechas tuvieron pendientes positivas no distintas de uno, y ordenadas al origen no distintas de cero (Tabla 1). Sin embargo, en tres fechas para los 9 meses y en dos fechas para los 12 meses las regresiones no fueron significativas debido a la baja variabilidad en los datos (Tabla 1). En cuatro de los cinco casos, el $\mathrm{R}^{2}$ para el modelo de 12 meses fue más alto que para el modelo de 9 meses (Tabla 1). Por último, cuando consideramos todas las fechas juntas, las regresiones fueron significativas, las pendientes no difirieron de uno, y las ordenadas al origen no difirieron de cero (Tabla 1; Figuras 4a y b). La varianza explicada fue más alta para el modelo de 12 meses (Figura 4a), la pendiente fue más próxima al valor 1, y la ordenada al origen más próxima al valor cero (Tabla 1). Todos estos resultados, conjuntamente con la comparación de los RCME, indican que el modelo de 12 meses "carga ganadera $=0.017 *$ frecuencia de deposiciones" es el más conveniente (ver Figuras $3 b$ \& $4 b$; Tabla 1)

\section{DisCUSIÓN}

Los resultados obtenidos nos permitieron desarrollar un modelo de estimación de carga ganadera localizada para las Sierras Grandes de Córdoba utilizando la frecuencia de deposiciones. El tipo de relación obtenida permite corroborar que la cantidad de 
Tabla 1. Varianza explicada por las regresiones de validación entre la carga informada y la carga estimada con los modelos para 9 y 12 meses previos utilizando diferentes conjuntos de datos externos correspondientes a cinco fechas diferentes. Los valores de ordenada al origen y pendiente de las regresiones de validación corresponden al cálculo realizado con los cinco conjuntos de datos agrupados. FD significa frecuencia de deposiciones. Los asteriscos indican relaciones estadísticamente significativas $(P<0.001)$.

Table 1. Explained variability of regression models used for validation among mean informed paddock stocking rates and the estimated paddock stocking rate by using the 9 and 12 polled data models of external dataset corresponding of five dates. Details in legend in Spanish.

\begin{tabular}{|c|c|c|}
\hline $\begin{array}{l}\text { Tiempo de integración } \\
\text { previo }\end{array}$ & 9 meses & 12 meses \\
\hline Modelo estimativo & $\begin{array}{c}\text { Carga (UG/ } \\
\text { ha) }=0.0184^{*} \mathrm{FD}\end{array}$ & $\begin{array}{c}\text { Carga (UG/ } \\
\text { ha) }=0.0170^{*} \mathrm{FD}\end{array}$ \\
\hline Conjuntos de datos & $\mathrm{R}^{2}$ & $\mathrm{R}^{2}$ \\
\hline Marzo 2007 (n=7) & 0.07 & $0.59^{*}$ \\
\hline Setiembre $2007(n=7)$ & 0.36 & 0.28 \\
\hline Enero $2008(\mathrm{n}=7)$ & 0.26 & 0.39 \\
\hline Agosto 2008 (n=11) & $0.79^{*}$ & $0.86^{*}$ \\
\hline Marzo 2009 (n=18) & $0.55^{*}$ & $0.73^{*}$ \\
\hline $\begin{array}{l}\text { Todos agrupados } \\
(\mathrm{n}=50)\end{array}$ & $0.51^{*}$ & $0.61^{*}$ \\
\hline Ordenada al origen & $-0.0020 \pm 0.0389$ & $-0.0012 \pm 0.0319$ \\
\hline Pendiente & $0.822 \pm 0.235$ & $0.888 \pm 0.208$ \\
\hline
\end{tabular}

deposiciones presentes en un determinado lugar se relaciona de manera lineal con el tiempo que los animales permanecen en ese lugar, tal como fuera observado por White et al. (2001) en un tipo de sistema muy diferente. En este sentido, los resultados son alentadores ya que sugieren que un tipo de metodología muy similar puede ser ajustada para diferentes ecosistemas y bajo diferentes condiciones de manejo ganadero. Asimismo, la relación lineal encontrada confirma que es adecuado evaluar los patrones de distribución del ganado a través de las deposiciones, tal como se ha hecho en otros trabajos en distintos sistemas (Cingolani et al. 2002, 2003; Tate et al. 2003; Kohler et al 2008). Nosotros, sin embargo, avanzamos un paso más en relación a otros trabajos al estimar las cargas bovinas localizadas para los sitios evaluados. Estos valores de carga localizada pueden ser de mucha utilidad a la hora de evaluar efectos puntuales del ganado bovino sobre otros componentes del ecosistema. Además, el modelo desarrollado permite calcular cargas de potreros enteros en los cuales se desconoce el manejo, siempre que el muestreo de las deposiciones sea adecuado y representativo.

La frecuencia de deposiciones reflejó de forma adecuada la carga ganadera localizada de seis a 15 meses previos a la medición. No obstante, este indicador estimó mejor la carga del año previo a la medición (12 meses) que la de otros períodos. El principal problema de los períodos menores o mayores a 12 meses fue que las ordenadas al origen se hicieron cada vez más negativas. Esto probablemente se deba a que al incluir períodos menores o mayores a la unidad temporal de manejo (año completo), los potreros con sistema de pastoreo rotativo hayan sesgando las regresiones. Por otro lado, para los períodos mayores a 12 meses, la varianza explicada por los modelos tuvo una tendencia a incrementarse. Esto puede significar que las deposiciones en el suelo reflejan períodos más prologados de carga (2 ó más años). En las Sierras de Córdoba, Vaieretti (2010) registró valores de descomposición de 13 a 15\% de pérdida de peso seco durante la época estival (enero a marzo), y de 8 a 10\% durante la época otoño-invernal (abril a julio). Estos resultados indican que las deposiciones efectivamente permanecen por más de un año en el suelo. Debido a que los potreros estudiados tuvieron un manejo constante durante varios años, esto no fue un obstáculo para que el modelo seleccionado resulte adecuado para estimar la carga de un único año previo, aunque hay que considerar que la estimación podría resultar sesgada si se producen cambios bruscos en el manejo de un año a otro. En síntesis, dentro del período de tiempo que pudimos considerar, el modelo "carga ganadera $=0.017$ * frecuencia de deposiciones", resultó el mejor, y refleja adecuadamente la carga ganadera de los 12 meses previos.

Diversos factores podrían afectar la tasa de deyecciones y/o su tasa de descomposición, disminuyendo la eficacia de los modelos estimativos. Sin embargo, es conocido que la tasa de deyecciones del ganado bovino se mantiene relativamente estable en diferentes ambientes, ya que los rumiantes deben necesariamente mantener las tasas de consumo de fibra en la dieta (Provenza et al. 2003) y, como consecuencia de ello, defecan a una tasa relativamente estable (van Soest 1994). La tasa de descomposición de las heces, en cambio, depende de numerosos factores como la calidad del forraje disponible para el herbívoro y las condiciones microambientales (humedad y de temperatura del micrositio) (Aerts 1997). Sin embargo, en el área de estudio, Vaieretti et al (2010) no encontraron diferencias en la descomposición de las heces bovinas entre diferentes tipos de parche de vegetación; esto sugiere que el modelo no está sesgado en relación al factor microambiental. Por otro lado, los antiparasitarios comerciales utilizados en el manejo sanitario bovino pueden afectar 
tanto la tasa de deyecciones bovinas, como la posterior tasa de descomposición (Sommer \& Bibby 2002). No obstante, su efecto es muy puntual (Iglesias et al. 2006) en relación a los períodos considerados en este estudio, y -además- los antiparasitarios se aplican una sola vez al año. Si bien la residualidad elevada de los antiparasitarios en las heces hace que los organismos descomponedores sensibles se vean afectados en su actividad (Sommer \& Bibby 2002), estos efectos no se ven reflejados en la desaparición de las heces en el suelo (Madsen et al. 1990).

Las diferencias en el manejo del pastoreo también pueden afectar las estimaciones (White et al. 2001; Tate et al. 2003). Nosotros encontramos diferencias entre los potreros rotativos estacionales según el momento en que se hizo la evaluación de frecuencia de deposiciones respecto a la entrada o salida de los animales. Esto se debe a que en los potreros con manejo rotativo del pastoreo, las deposiciones ocurren sólo en determinados momentos del año, mientras que la descomposición sigue ocurriendo por más que no haya animales. Es por ello que cuando la medición se realizó hacia el final del período de descanso del potrero, se subestimó la carga ganadera. Por el contrario, cuando la medición se realizó hacia el final del período de pastoreo (o inicios del período de descanso) el modelo sobreestimó la carga ganadera. Si bien la varianza explicada por el factor de manejo no fue muy alta, es importante tener en cuenta este efecto al aplicar el modelo. En potreros con manejo continuo del pastoreo, las estimaciones de carga resultan menos sesgadas. Esto implica que si se utiliza el modelo sin conocimiento del manejo puede haber algún sesgo en las estimaciones, y sería conveniente medir la frecuencia de deposiciones al menos dos veces en el año, espaciadas entre sí, y hacer un promedio de las cargas estimadas. En casos de conocerse el manejo, si es continuo las mediciones se pueden hacer en cualquier momento, y si es rotativo, lo más adecuado sería hacer las mediciones hacia la mitad del período de descanso o de pastoreo.

La validación del modelo en otros potreros de la misma región nos permitió confirmar la utilidad del modelo obtenido y brindar mayor potencia a los resultados (Sørensen 1990). Si bien la validación se realizó a escala de potrero completo y no de parcela, como hubiera sido ideal, nuestro trabajo mejora mucho lo realizado en otros estudios similares, en los cuales se utiliza un solo potrero para la validación (Senft et al. 1983). El modelo estimativo obtenido en el presente trabajo fue elaborado con el objetivo de ser utilizado en el piso superior de las Sierras Grandes de Córdoba. Si bien el ajuste de los modelos fue realizado en una porción relativamente pequeña del Parque Nacional y Reserva Nacional aledaña (siete potreros, 3392 ha en total), la validación permitió extenderlo a un área bastante más grande (18 potreros, en total 9771 ha). No se ha realizado una validación en otras zonas del piso superior de las Sierras Grandes. Sin embargo, en la mayor parte de la zona alta de este cordón montañoso la vegetación y las condiciones climáticas se mantienen similares, ya que las zonas de mayor o menor altitud (y por ende distinta temperatura media) a la considerada en este estudio ocupan poca superficie (Cabido 1985; Cingolani et al. 2008b). Asimismo, el tipo y manejo de los animales es similar en el resto de las Sierras Grandes. Por esta razón creemos que el modelo es aplicable al resto de esta región. Por otro lado, las variaciones interanuales de precipitación y temperatura pueden afectar de manera sensible a la tasa de descomposición de las heces en el suelo (Aerts 1997) y, del mismo modo, pueden limitar la utilización de los modelos a otros años con diferentes condiciones meteorológicas. Sin embargo, la validación se realizó considerando diferentes fechas, que abarcaron tres años diferentes; esto sugiere que el modelo es lo suficientemente robusto como para ser utilizado de manera independiente de las variaciones meteorológicas interanuales. Es de destacar que de los tres años que incluyen la validación, uno fue cálido y seco (2006/2007), otro frío y húmedo $(2007 / 2008)$, y el último templado y seco (2008/2009; Colladón et al. 2010).

Consideramos que es posible utilizar modelos similares al obtenido en el presente estudio para cuantificar la intensidad localizada de uso ganadero en otras regiones y momentos. Sin embargo, para cuantificar cargas localizadas y teniendo en cuenta que tanto las condiciones climáticas como el manejo ganadero puede afectar los resultados, es fundamental realizar una corrección del modelo mediante un análisis al menos a escala de potrero. Finalmente, es necesario remarcar que el modelo tal cual se presenta en este trabajo únicamente es válido cuando la presencia de deposiciones bovinas es contabilizada en un cuadrado de $30 \times 30 \mathrm{~cm}$, pero se podría corregir para otros tamaños de cuadrado. 
Agradecimientos: A las autoridades del PNQC y a la DRC (APN), especialmente a G. Jaacks y M. Ferioli. A G. Raggio de la Estancia Santo Tomás. Este trabajo se realizó mediante una beca doctoral de CONICET y financiado con subsidio PIP-CONICET Nro. 6196/2006 y 112-20080101458 y del MINCyT (Prov. de Córdoba).

\section{REFERENCIAS}

Adler, PB; DA Raff \& WK Lauenroth. 2001. The effect of grazing on the spatial heterogeneity of vegetation. Oecologia, 128:465-479.

AERTS, R. 1997. Climate, leaf litter chemistry and leaf litter decomposition in terrestrial ecosystems: a triangular relationship. Oikos, 79:439-449.

BAILEY, DW. 1995. Daily selection of feeding areas by cattle in homogeneous and heterogeneous environments. Appl. Anim. Behav. Sci., 45:183-200.

Bailey, DW; JE Gross; EA Laca; LR Rittenhouse; MB COUGHenOUR; ET AL. 1996. Mechanisms that result in large herbivore grazing distribution patterns. J. Range. Manage., 49:386-400.

Barnes, MK; BE Norton; M Maeno \& JC Malechek. 2008. Paddock Size and Stocking Density Affect Spatial Heterogeneity of Grazing. Range. Ecol. Manage., 61:380-388.

Bertiller, MB \& JO Ares. 2008. Sheep spatial grazing strategies at the arid Patagonian Monte, Argentina. Range. Ecol. Manage., 61:38-47.

CABido, M. 1985. Las comunidades vegetales de la Pampa de Achala. Sierras de Córdoba, Argentina. Doc. Phytosociologiques, 9:431-443.

Cingolani, A; J Anchorena; S Stoffella \& M Collantes. 2002. A landscape-scale model for optimal management of sheep grazing in the Magellanic steppe. Appl. Veg. Sci., 5:159-166.

Cingolani, A; M Cabido; D Renison \& V Solís-NefFa. 2003. Combined effects of environment and grazing on vegetation structure in Argentine granite grasslands. J. Veg. Sci., 14:223-232.

Cingolani, A; D Renison; M ZaK \& M Cabido. 2004. Mapping vegetation in a heterogeneous mountain using Landsat data: an alternative method to define and classify land-cover units. Rem. Sens. Environ., 92:84-97.

Cingolani, AM; I Noy-MeIr \& S Díaz. 2005. Grazing effects on rangeland diversity: A synthesis of contemporary models. Ecol. Appl., 15:757-773.

Cingolani, AM; I Noy-Meir; D Renison \& MD Cabido. 2008a. La ganadería extensiva, ¿es compatible con la conservación de la biodiversidad y de los suelos? Ecología Austral, 18:253-271.

Cingolani, AM; D Renison; PA Tecco; DE Gurvich \& M CABIDO. 2008b. Predicting cover types in a mountain range with long evolutionary grazing history: a GIS approach. J. Biogeogr., 35:538-551.

Cingolani, AM; MV VaieretTI; DE GurVich; MA Giorgis \& M CABIDO. 2010. Predicting alpha, beta and gamma plant diversity from physiognomic and physical indicators as a tool for ecosystem monitoring. Biol. Conserv., 143:2570-2577.

Cocimano, M; A Lange \& E Menvielle. 1975. Estudio sobre equivalencias ganaderas. Producción Animal, 4:161-190.

Colladón, L; GSFelici \& IPAZos. 2010. Anuario Pluviométrico 2005/06-2009/10. Cuenca del RíoSan Antonio. Sistema del Río Suquia-Provincia de Córdoba. INA y CRSA.

Dimander, SO; J Höglund \& PJ Waller. 2003. Disintegration of dung pats from cattle treated with the ivermectin anthelmintic bolus, or the biocontrolv agent Duddingtonia flagrans. Acta Vet. Scand., 44:171-180.
FUHLENDORF, SD \& DMENGLE. 2001. Restoring Heterogeneity on Rangelands: Ecosystem Management Based on Evolutionary Grazing Patterns. BioScience, 51:625-632.

Grigera, G; M Oesterheld \& F Pacín. 2007. Monitoring forage production for farmers' decision making. Agric. Syst., 94:637-648.

Iglesias, L; C SAumell; A FernándeZ; L Fusé; A LifschitZ; ET AL. 2006. Environmental impact of ivermectin excreted by cattle treated on dung fauna and degradation of faeces on pasture. Parasitol. Res., 100: 93-102.

KEMP, DR \& DL MichaLK. 2007. Towards sustainable grassland and livestock management. J. Agr. Sci., 145:543-564.

Kohler, F; F Gillet; S Reust; HH Wagner; F Gadallah; et AL. 2006. Spatial and seasonal patterns of cattle habitat use in a mountain wooded pasture. Landscape Ecol., 21: 281-295.

Madsen, M; B Overgaard Nielsen; P Holter; OC Pedersen; J BROCHNER JESPERSEN; ET AL. 1990. Treating cattle with Ivermectin: Effects on the fauna and decompsition of dung pats. J. Appl. Ecol., 27:1-15.

Provenza, FD; JJ Villalba; LE Dziba; SB Atwood \& RE BANNE. 2003. Linking herbivore experience, varied diets, and plant biochemical diversity. Small Rumin. Res., 49: 257-274.

Senft, RL; LR Rittenhouse \& RG Woodmansee. 1983. The use of regression models to predict spatial patterns of cattle behavior. J. Range Manage., 36:553-557.

Senft, RL; MB Coughenour; DW BaILeY; LR RitTenhouse; OE SALA; ET AL. 1987. Large herbivore foraging and ecological hierarchies. BioScience, 37:789-799.

SOMmer, C \& BM BibBy. 2002. The influence of veterinary medicines on the decomposition of dung organic matter in soil. Eur. J. Soil Biol., 38:155-159.

SøREnsEn, JT. 1990. Validation of livestock herd simulation models: a review. Livest. Prod. Sci., 26:79-90.

Swain, DL; MA Friend; GJ Bishop-HuRley; RN Handcock \& T WARK. 2011. Tracking livestock using global positioning systems - are we still lost? Anim. Prod. Sci., 51:167-175.

SWIFT, MJ; OW HeAl \& JM ANDERSON. 1979. Decomposition in Terrestrial Ecosystems. Blackwell, Oxford.

TATE, KW; ER Atwill; NK McDougald \& MR George. 2003. Spatial and temporal patterns of cattle feces deposition on rangeland. J. Range Manage., 56:432-438.

Teich, I; AM Cingolani; D Renison; I Hensen \& MA Giorgis. 2005. Do domestic herbivores retard Polylepis australis Bitt. Woodland recovery in the mountains of Córdoba, Argentina? For. Ecol. Manage., 219:229-241.

VAIERETTI, MV. 2010. Estructura de la vegetación y dinámica de la descomposición: el rol de la herbivoría en pastizales de altura bajo uso ganadero (Córdoba, Argentina). Tesis doctoral. FCEFyN-UNC. Córdoba. Argentina.

Vaieretti, MV; AM Cingolani; N Pérez HarguindeguY; DE GuRvich \& M CABIDO. 2010. Does decomposition of standard materials differ among grassland patches maintained by livestock? Austral Ecol., 35:935-943.

VAN SOEST, PJ. 1994. Nutritional Ecology of the Ruminant. Cornell University Press. USA.

VON MüLler, A. 2011. Factores ecológicos y de manejo en la selección de hábitat de herbívoros domésticos en las Sierras Grandes de Córdoba. Tesis doctoral. FCEFyN-UNC. Córdoba. Argentina.

White, SL; RE SHeFfieLd; SP WASHBuRn; LD KING \& JT GREEN. 2001. Spatial and time distribution of dairy cattle excreta in an intensive pasture system. J. Environ. Qual., 30:2180-2187. 


\section{INFORMACIÓN SUPLEMENTARIA}

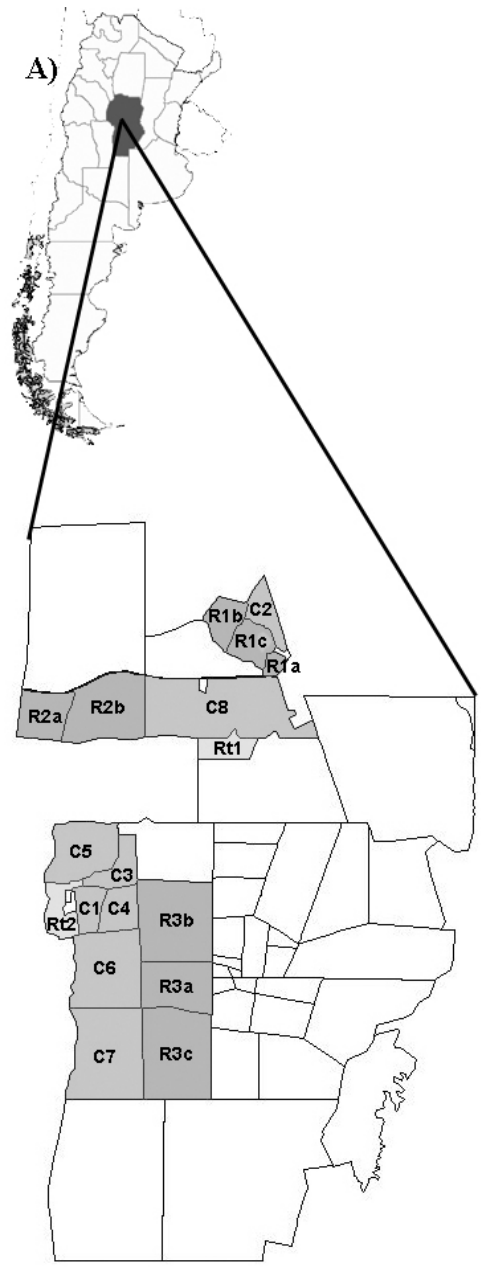

Parque Nacional Quebrada del Condorito
B)

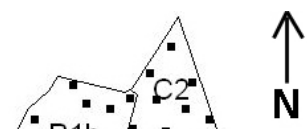

N
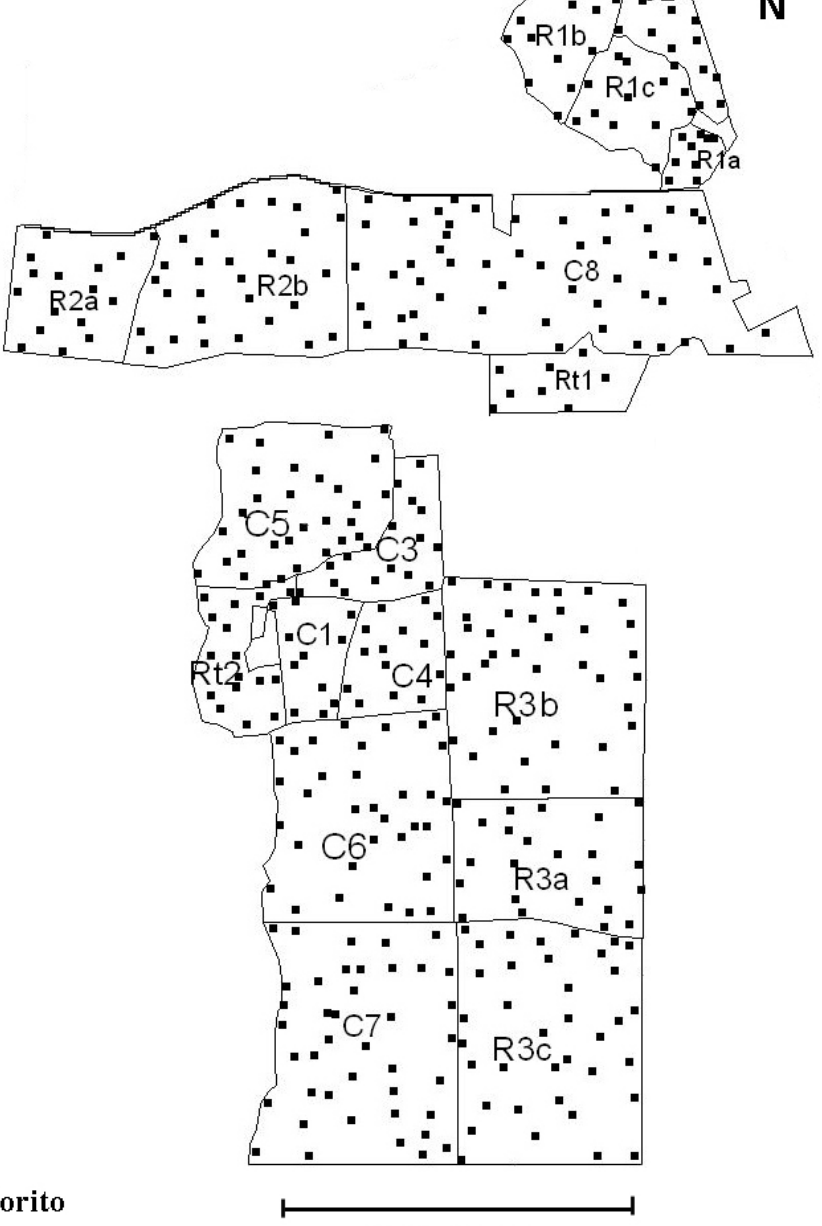

5 kilometros

Anexo I. a) Ubicación del Parque Nacional Quebrada del Condorito en la Provincia de Córdoba (Argentina) y los potreros de estudio dentro del Parque. b) Ampliación de los potreros y ubicación de las parcelas de estudio de 1 ha, representadas por los cuadrados pequeños dibujados a escala. La letra " $\mathrm{C}$ " indica potreros con sistema de pastoreo continuo; y la letra " $\mathrm{R}$ " indica potreros con sistema de pastoreo rotativo estacional. En los potreros rotativos el mismo número indica potreros pertenecientes a una misma unidad de rotación y la letra el orden de pastoreo. Los potreros

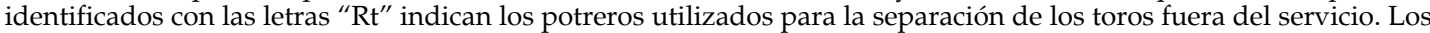
potreros R2a y R2b pertenecen a la Estancia Santo Tomás, en la Reserva Nacional, y los restantes al Parque Nacional propiamente dicho.

Appendix I. a) Location of Quebrada del Condorito National Park in the province of Córdoba (Argentina); and paddocks included in the present study within the Park. b) Detail of the study paddocks and plots of 1 ha, represented by the scale dimensioned squares. The paddock names which begin with a " $\mathrm{C}$ " indicate continuous grazing systems; the paddock names which begin with an " $R$ " indicate seasonal rotational grazing systems. In the rotational grazing paddocks, the same number indicates units belonging to the same grazing system and the letter indicates grazing order. Paddocks identified with "Rt" were used to isolate bulls out of mating season. Paddocks R2a and R2b belong to the private settlement Santo Tomás, in the National Reserve, and all of the other paddocks belong to the National Park. 
Anexo II. Características y manejo de los potreros utilizados para el ajuste (A) y validación (V) de los modelos para estimar la carga ganadera localizada a partir de la frecuencia de deposiciones fecales bovinas.

Appendix II. Characteristics and management of study paddocks used to model assessment (A) and model validation (V) to estimate localized stocking rate from cattle dung frequency.

\begin{tabular}{|c|c|c|c|c|c|c|c|c|c|}
\hline \multirow{3}{*}{ Potrero $^{1}$} & \multirow{3}{*}{$\begin{array}{l}\text { Período de } \\
\text { pastoreo }\end{array}$} & \multirow{3}{*}{$\begin{array}{l}\text { Superficie total } \\
\text { (ha) }\end{array}$} & \multirow{3}{*}{$\begin{array}{l}\text { Número de } \\
\text { Parcelas }\end{array}$} & \multirow{3}{*}{$\begin{array}{c}\text { Carga media } \\
\text { anual (UG/ha) }{ }^{2}\end{array}$} & \multicolumn{5}{|c|}{ Frecuencia de deposiciones $^{3}$} \\
\hline & & & & & Mar & Set & Ene & Jul/Ago & Mar \\
\hline & & & & & 2007 & 2007 & 2008 & 2008 & 2009 \\
\hline $\mathrm{C} 1$ & Anual & 199 & 11 & 0.00 & - & - & - & $\mathrm{V}$ & $\mathrm{V}$ \\
\hline $\mathrm{C} 2$ & Anual & 226 & 14 & 0.09 & $\mathrm{~V}$ & $\mathrm{~V}$ & V & A & V \\
\hline $\mathrm{C} 3$ & Anual & 258 & 16 & 0.16 & - & - & - & V & V \\
\hline $\mathrm{C} 4$ & Anual & 266 & 14 & 0.12 & - & - & - & V & $\mathrm{V}$ \\
\hline C5 & Anual & 608 & 30 & 0.18 & - & - & - & V & $\mathrm{V}$ \\
\hline C6 & Anual & 875 & 34 & 0.21 & - & - & - & $\mathrm{V}$ & $\mathrm{V}$ \\
\hline C7 & Anual & 1074 & 41 & 0.16 & - & - & - & $\mathrm{V}$ & V \\
\hline C8 & Anual & 1453 & 55 & 0.13 & V & V & V & A & V \\
\hline R1a & Dic-ene & 70 & 9 & 0.12 & $\mathrm{~V}$ & V & V & A & V \\
\hline R1b & ene-jun & 239 & 12 & 0.19 & V & V & V & A & V \\
\hline R1c & jul-dic & 256 & 13 & 0.16 & V & V & V & A & V \\
\hline R2a & Jul-nov & 391 & 16 & 0.13 & V & V & V & A & V \\
\hline $\mathrm{R} 2 \mathrm{~b}$ & nov-jun & 757 & 30 & 0.18 & V & V & V & A & V \\
\hline R3a & Jun & 573 & 23 & 0.03 & - & - & - & V & V \\
\hline $\mathrm{R} 3 \mathrm{~b}$ & Jul-sep & 1020 & 39 & 0.12 & - & - & - & V & V \\
\hline R3c & Abr-may & 1035 & 37 & 0.06 & - & - & - & V & V \\
\hline Rt1 & may-nov & 172 & 8 & 0.02 & - & - & - & V & V \\
\hline Rt2 & may-nov & 242 & 17 & 0.04 & - & - & - & $\mathrm{V}$ & $\mathrm{V}$ \\
\hline
\end{tabular}

1 En los potreros rotativos el mismo número indica potreros pertenecientes a una misma unidad de rotación y la letra el orden de pastoreo. Los potreros identificados con las letras " $\mathrm{Rt}^{\prime}$ indican los potreros utilizados para la separación de los toros. Los potreros R2a y R2b pertenecen a la Estancia Santo Tomás, en la Reserva Nacional, y los restantes al Parque Nacional propiamente dicho.

2 Carga media anual bovina en todo el período de estudio incluyendo la validación, desde marzo 2006 hasta febrero 2009.

3 Fechas en las que se midió la frecuencia de deposiciones en las parcelas de los diferentes potreros. A: potreros y fechas de medición utilizados para el ajuste de los modelos, V: utilizados para la validación de los modelos.

1 In the rotational grazing paddocks, the same number indicates units belonging to the same grazing system and the letter indicates grazing order. Paddocks identified with " $\mathrm{Rt}$ " were used to isolate bulls. Paddocks R2a and R2b belong to the private settlement Santo Tomás, in the National Reserve, and the other paddocks belong to the National Park. 2 Mean annual stocking rate of cattle during the whole study period including validation, from March 2006 to February 2009.

3 Months in which dung frequency was measured within the plots from the different paddocks. A: paddocks and months used for model estimation; and V: paddocks and months used for model validation. 